\title{
ULTRAVIOLET OBSERVATIONS OF CLASSICAL NOVAE IN OUTBURST
}

\author{
Angelo Cassatella(1) and Rosario Gonzalez-Riestra
}

ESA IUE Observatory, Villafranca, Spain

(1) Istituto Astrofisica Spaziale, CNR, Frascati, Italy

\section{Introduction}

More than a dozen classical novae in outburst have been observed with the International Ultraviolet Explorer since its launch in 1978 . These data represent a crucial breakthrough in understanding several aspects of the classical novae phenomenon including nucleosynthesis, the energetics of the explosion, the dynamics of the mass ejection, and the processes of dust formation. Algo they are important to gain insight on the nature of the white dwarf components and then on the evolutionary scenario of these binary systems.

The UV emission lines include important transitions from several species such as $\mathrm{He}, \mathrm{C}, \mathrm{N}$ and $O, \mathrm{Ne}, \mathrm{Mg}$, and $\mathrm{Al}$ which, whenever symultaneous optical data were available, have lead to a detailed knowledge of the physical conditions in the nebular phases and to the determination, with unprecedented accuracy, of the chemical abundances in the ejecta (see e.g.: Stickland et al. 1981; Williams et al. 1985; Snijders et al. 1987). Precise abundance determinations provide crucial constraints on nucleosinthesis during TNR as well as on the efficiency of diffusion and mixing processes. The ultraviolet range provides also effective means to determine the value of the reddening from the strength of the $2200 \mathrm{~A}$ dust feature or from the emission line ratios; an accurate determination of this parameter from the UV is essential, for example, to determine the energy budget of novae and the temperature of the remnants.

One of the major acheivements of ultraviolet plus simultaneous ground based observations is the determination of the bolometric light curves for V1668 Cyg 1978 (Stickland et al. 1981), v1370 Aq1 1982 (Snijders et al. 1981) and RS Oph (Snijders 1986), information before available only for FH Ser, after the pioneering work of Gallagher and Code (1974). Bolometric light curves for a few more novae are being analysed by different groups. The results so far available represent a gtrong argument in favour of thermonuclear runaway on a white dwarf companion as the driving mechanism of the outbursts. The mass of the white dwarf components can be directly estimated from the plateau in luminosity appearing after maximum, when novae radiate near the Eddington limit, provided the distance can be accurately evaluated. For the recurrent nova RS Oph, for example, it was possible to establish that the plateau in luminosity was consistent with the Eddington luminosity of a massive white dwarf (Snijders 1986), providing then a strong constraint to the evolutionary scenario of recurrent novae. Conversely, if the distance is unknown, it can be estimated from the plateau luminosity, as done for V1668 Cyg 1978 by Stickland et al. (1981). Decisive constraints on the energetics of novae can be obtained from extragalactic novae for which a multifrequency monitoring programme has been carried out, such as $N$ LMC 1988\#1. 
Ultraviolet observations have provided the first observational evidence for element depletion during a nova outburst produced by dust formation. As reported by Snijders et al. (1987) the elements $\mathrm{C}, \mathrm{O}, \mathrm{Mg}, \mathrm{Si}$ and $\mathrm{Fe}$ were apparently depleted in the gas phase in consequence of grain formation in V1370 Aq1 1982 . In this nova the mass of the dust-phase was of the same order of that of the gas-phase.

A major result of ultraviolet observations is the discovery of a new class of novae, the ONeMg novae (Starrfield, Sparks and Truran 1986), in which very large overabundances of intermediate masg nuclei like neon, magnesium, oxygen and aluminium have been found (see snijders et al. 1984,1987 for V1370 Aql 1982 and Williams et al. 1985 for V693 Cra 1981).

Further information on recent results obtained through ultraviolet observations can be found in the reviews by starrfield (1986), Starrfield and Snijders (1987), Friedjung (1989) and Cassatella and Selvelli (1988). We will concentrate here on aspects which have not been considered before, i.e. on the time behaviour of the continuum energy distribution during the early development of novae. Our purposes are: to look for a correlation between the time development of the light curves in the UV and in the optical ranges; to analyse the information contained in the UV two-colour vB. time diagrams; to provide estimates for the temperature of the nova remnants as late as possible after the outburst.

\section{The UV light curves}

Interesting information can be derived from a comparative study of the UV light curves of classical novae. We will restrict ourgelves to those objects whose active phases were reasonably well covered by IUE observations. The data were retrieved from the IUE Uniform Low Dispersion Archive (ULDA, see Wamsteker et al. 1989), from the VILSPA data bank or have been taken as a part of our observing programmes. The following eight novae were selected: V1668 Cys 1978, V693 CrA 1981, GQ Mus 1983, PW Vul 1984\#1, QU Vul 1984\#2, OS And 1986, N LMC $1988 \# 1$ and N LMC 1988\#2. For each object we have measured the observed UV mean fluxes in two bands centered at 1455 A ( 30 A wide) and $2855 \mathrm{~A}$ (40 A wide). These bands represent fairly well the continuum, since contamination by emission lines is there generally negligible. The time behaviour of the observed UV mean fluxes for the slow nova PW Vul 1984\#1 ( $t_{3}=97$ days), the moderately fast novae V1668 Cyg $1978 \quad\left(t_{3}=23\right.$ days) and OS And $1986\left(t_{3}=22\right.$ days), and the fast nova $N$ CrA 1981 ( $t_{3}=12$ days) is shown in Fig. 1a for the 1445 A band and Fig. Ib for the for the $2855 \mathrm{~A}$ band. It clearly appears from the figure that the 2855 A fluxes peak systematically earlier in time than the 1455 A fluxes, trend which is confirmed by the full set of novae analysed. In addition, the time difference between the peaks in the two bands tends to decrease whith increasing $t_{3}$ : it is about 37 days for the slow nova PW Vul while it becomes virtually zero for the very fast nova V693 CrA. This behaviour, applicable to the full set of novae considered here, is also present when comparing the UV with the optical light curves. Let us consider the band at $1455 \mathrm{~A}$ and define $\Delta t=t(1455 \mathrm{~A})$ - $t$ (opt) as the time delay in days between the optical peak and the peak at 1455 A. The adopted values for $t_{3}, t(1455)$ and $t$ (opt) are reported in Table 1 . It clearly appears that the time lag $\Delta t$ is a function of $t_{3}$, being very small (virtually zero) for fast novae and large for slow novae, as shown in Fig. 2. The observations can be 


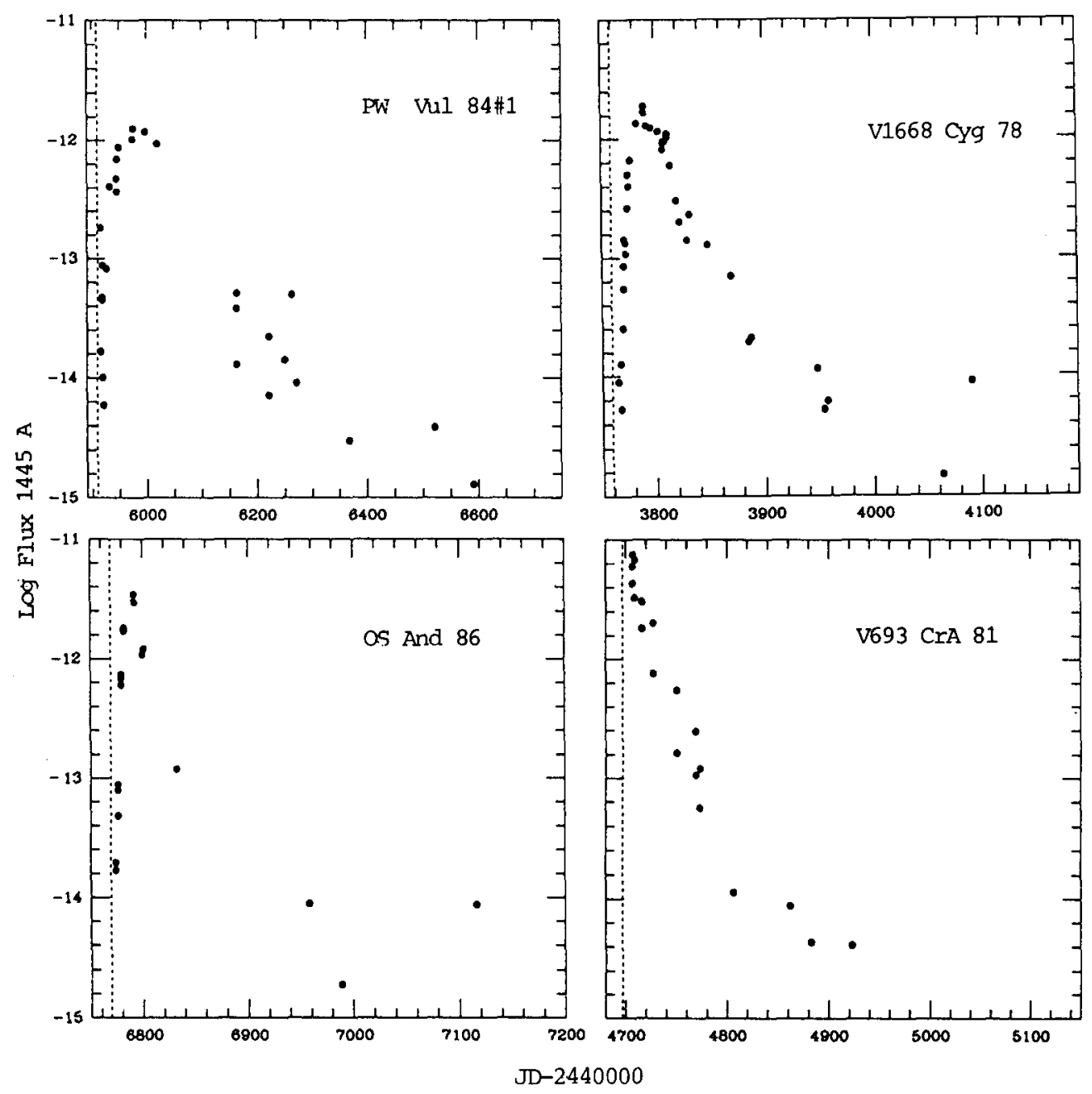

Fig. 1a Time behaviour of the ultraviolet flux at 1445 A for four novae monitored with IUE. Units for fluxes are $10^{-14} \mathrm{erg} \mathrm{cm}^{-2} \mathrm{~s}^{-1}$ $A^{-1}$. The vertical bar denotes the time of the optical outburst. 


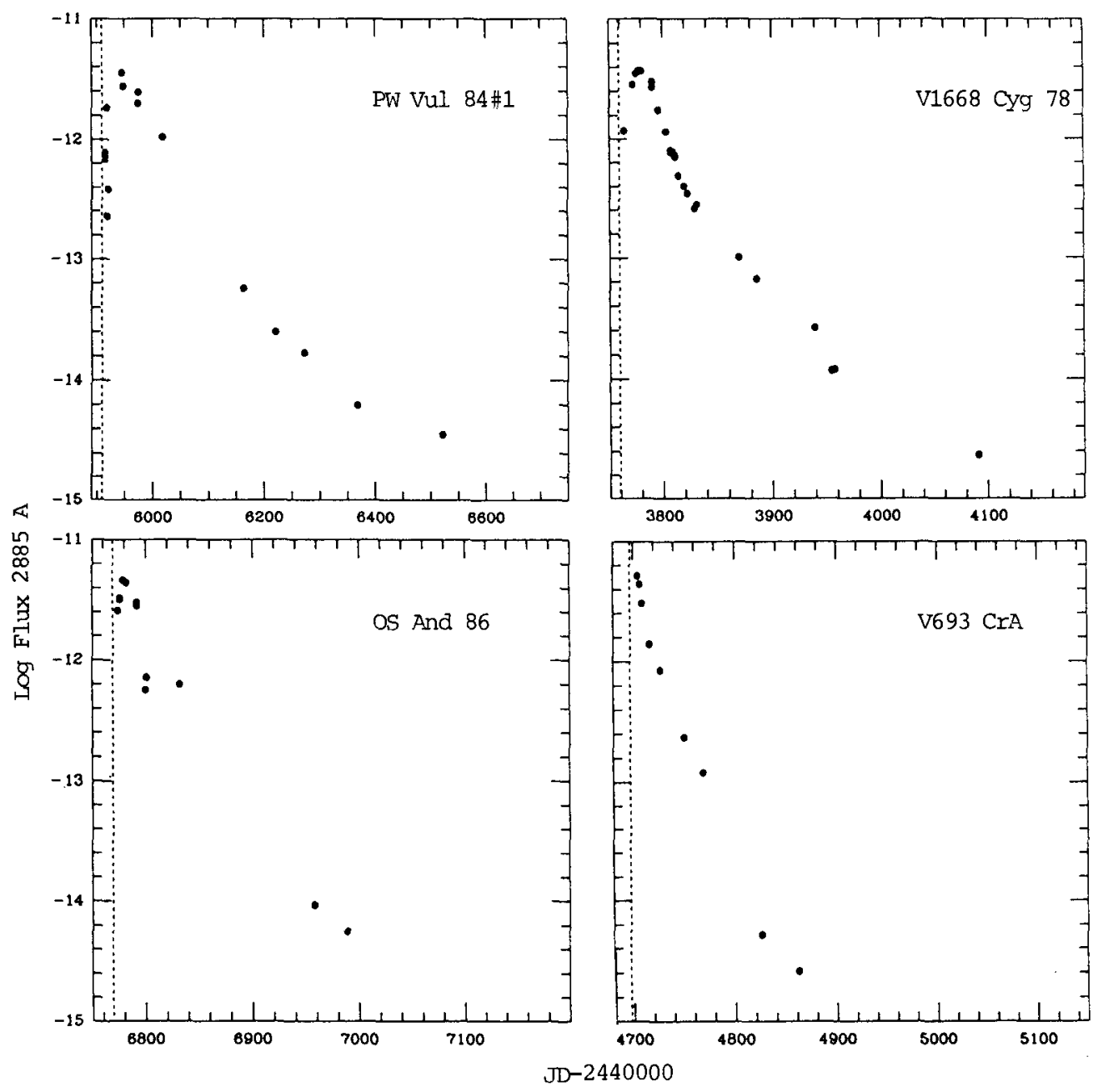

Fig. 1b Same as for Fig. 1a, at $2885 \mathrm{~A}$. 


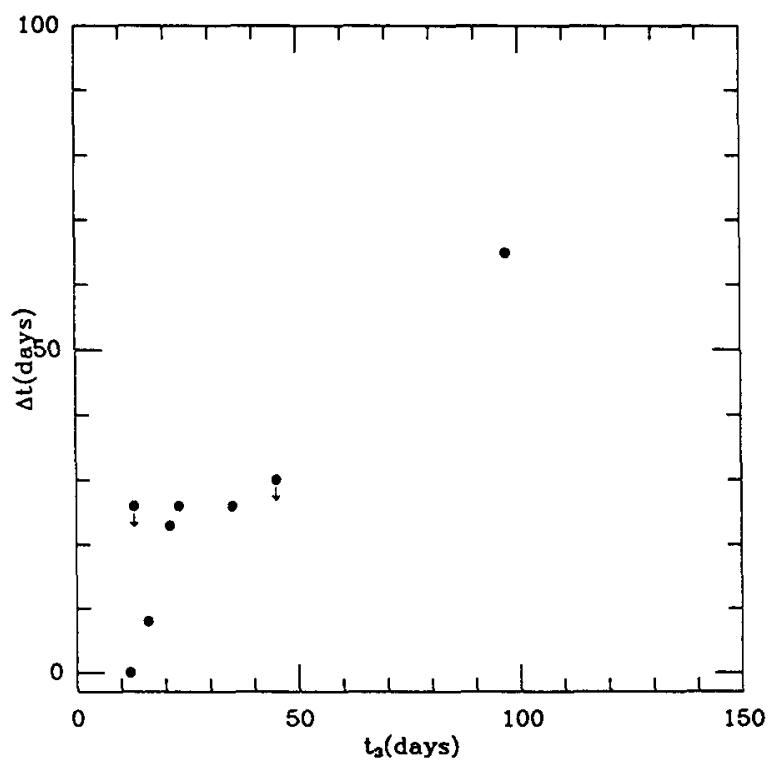

Fig. 2 Time lag $\Delta t$ between the optical outburst and the $U V$ maximum at 1445 as a function of the decline time $t_{3}$.

TABLE 1

\begin{tabular}{lccc}
\hline Object & $J_{0}-2440000$ & $\mathrm{t}_{3}$ & $\Delta \mathrm{t}$ \\
\hline V1668 Cyg '78 & 3759 & 23 & 26 \\
V693 CrA '81 & 4697 & 12 & $\sim 0$ \\
V1370 Aq1 '82 & 4998 & 13 & $<26$ \\
GQ Mus '83 & 5353 & 45 & $<30$ \\
PW Vul '84\#1 & 5909 & 97 & 65 \\
QU Vul '84\#2 & 6058 & 40 & - \\
OS And '86 & 6769 & 22 & 23 \\
N LMC '88\#1 & 7247 & 35 & $\sim 26$ \\
N LMC '88\#2 & 7447 & 16 & 8 \\
\hline
\end{tabular}



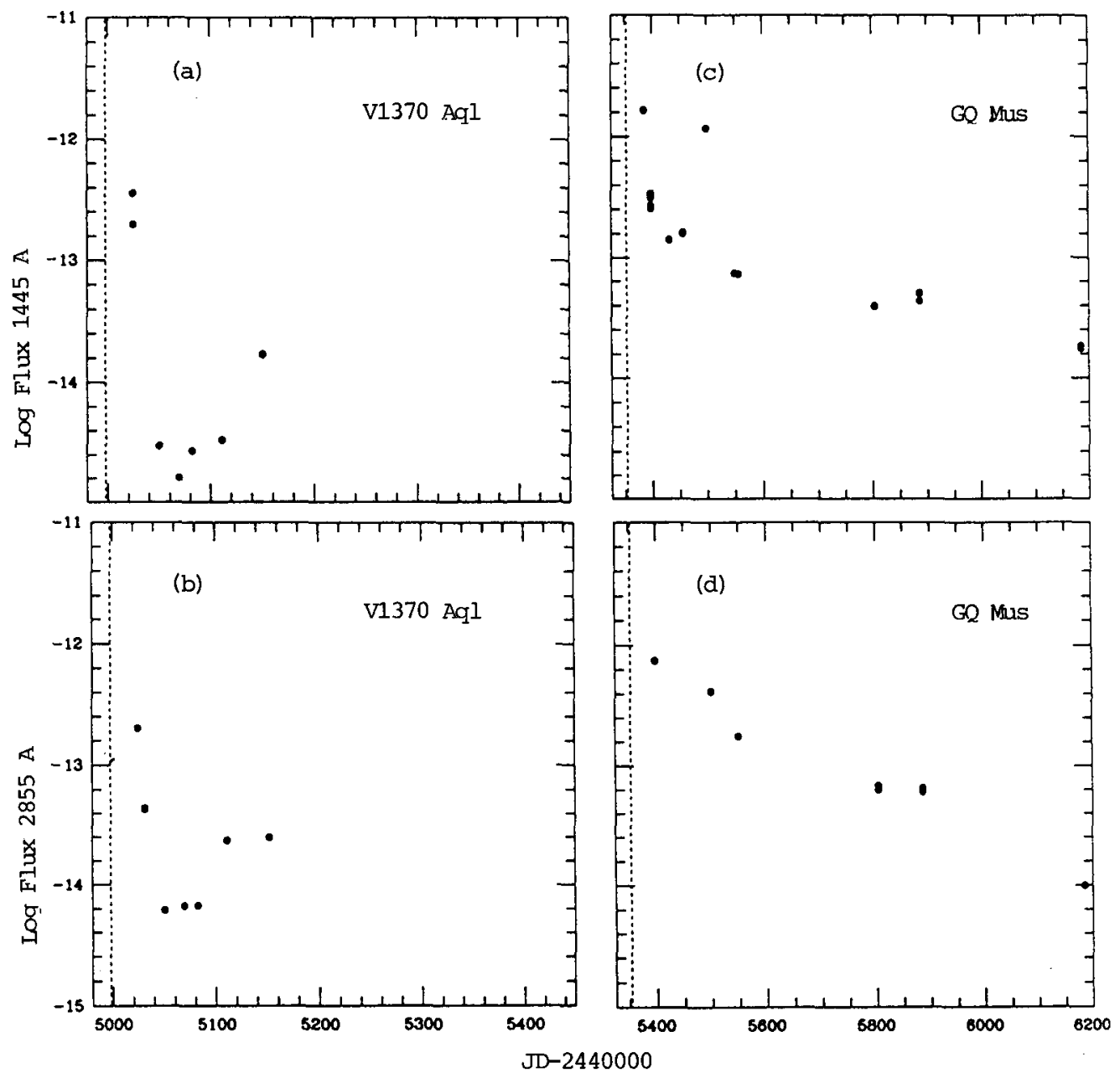

Fig. 3 UV light curves at 1445 A and 2885 A for two novae with a peculiar behaviour (compare with Fig. 1a,b): V1370 Aql 1982 (a,b) and GQ Mus $1983(c, d)$. 


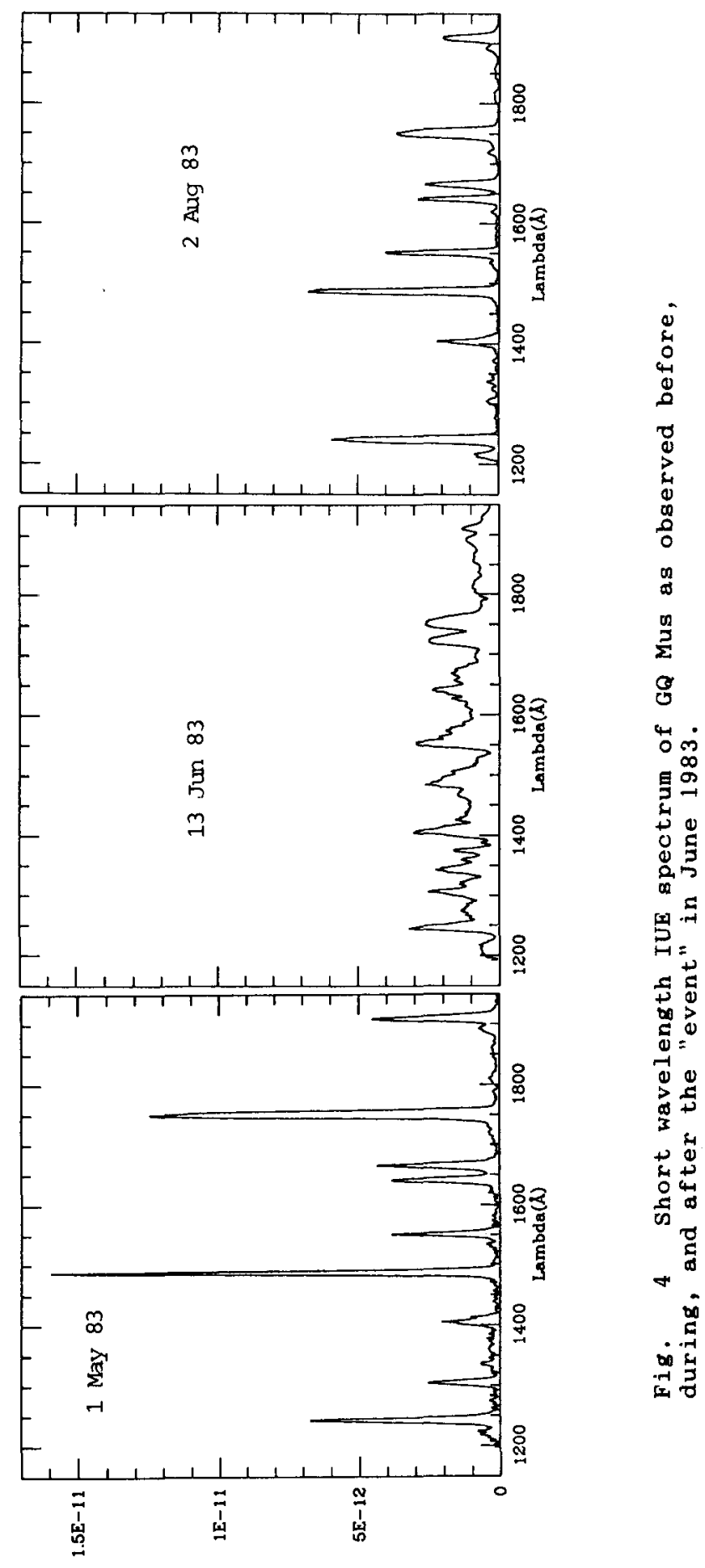


understood in terms of a pseudo-photosphere whose radius ghrinks while the object evolves at constant bolometric luminosity, causing the inner and hotter regions to be visible and producing a progressive shift of the maximum emigsivity towards shorter wavelengths as time increaseg. Neither the optical nor the ultraviolet maxima can then be taken as representative of the ejection time. A more realistic estimate of the ejection time is probably obtained from the time of the infrared peak (see Gehrz 1988 and references therein, and Wamsteker 1979 for the case of $\mathrm{N}$ Cyg 1975). Within this frame, the energy distribution is expected to change more rapidly for the brightegt novae i.e. for novae with shorter $t_{3}$ (Evans 1989; Bath and Harkness 1989), in agreement with the results in Fig. 2 .

The UV light curves show a smooth decline after maximum except for two objects of our sample: v1370 Aq1 1982, ghown in Fig. 3a, b, where a deep minimum appeared around day 77 , and GQ Mus, shown in Fig. $3 c, d$, where a secondary sharp and intense peak emission was observed in the 1455 A band (but not in the 2855 A band) near day 150. The dip observed in the UV light curve of V1370 Aql around day 77 has been interpreted in terms of absorption of radiation by dust internal to the nova shell, followed by re-emission in the infrared (Snijders et al. 1987). V1370 Aql 1982 is not the only nova among those considered here which has been suggested to contain dust, but is the only one in our sample in which the presence of dust has apparently lead to a temporary decrease of the UV fluxes during the decline phase. In GQ Mus, the spectra taken during the secondary maximum, compared to the spectra before and after the event around day 150, indicate a general fainting and broadening of the emission lines as well as a temporary decrease of the ionization level, as shown in Fig. 4. This behaviour, togheter with the observed broadenning of the emission lines was interpreted by Hassall et al. (1990) as the evidence of an increased mass transfer rate fron the secondary star or as a sudden mass ejection episode from the white dwarf triggered by a flare in the nuclear burning.

\section{Time evolution of the UV coloura}

The ultraviolet continuum of novae is due to a combination of nebular emission and of emission from a pseudo-photosphere. The relative importance of the two components depends on the stage of evolution of the object and on wavelength. In the earliest phases of the outburst optically thick emission from a cool pseudo-photosphere is dominant. As the envelope expands and radiation from the central source becomes harder, an increasing fraction of the envelope becomes photoionized and nebular continuum is emitted. During the nebular phase, the far ultraviolet continuum (e.g. at 1455 A) becomes dominated by emission from the pseudo-photosphere, while at longer wavelengths (e.g. 2855 A) it is largely due to Balmer recombination continuum (see Fig. 5 in stickland et al. 1981). The set-in of the nebular regime can be easily detected in the UV range when plotting the UV colour $C(1455-2855)=m(1455)-m(2855)$ as a function of time. In Fig. 5 we show the time behaviour of C(1455-2855) for v1668 Cyg 1978, N LMC 1988\#1, and OS And 1986. In these cases as well as in the case of V693 CrA (not shown), the UV continuum becomes progressively hotter during the early otburst development until the UV colour reaches a relative minimum (near day 51 for V1668 Cyg, day 57 for $N$ LMC 1988\#1, day 32 for os And, and day 85 for v693 CrA). After the minimum, the the UV colour reaches a relative maximum appearing redder for some time, until the original trend for the continuum to 


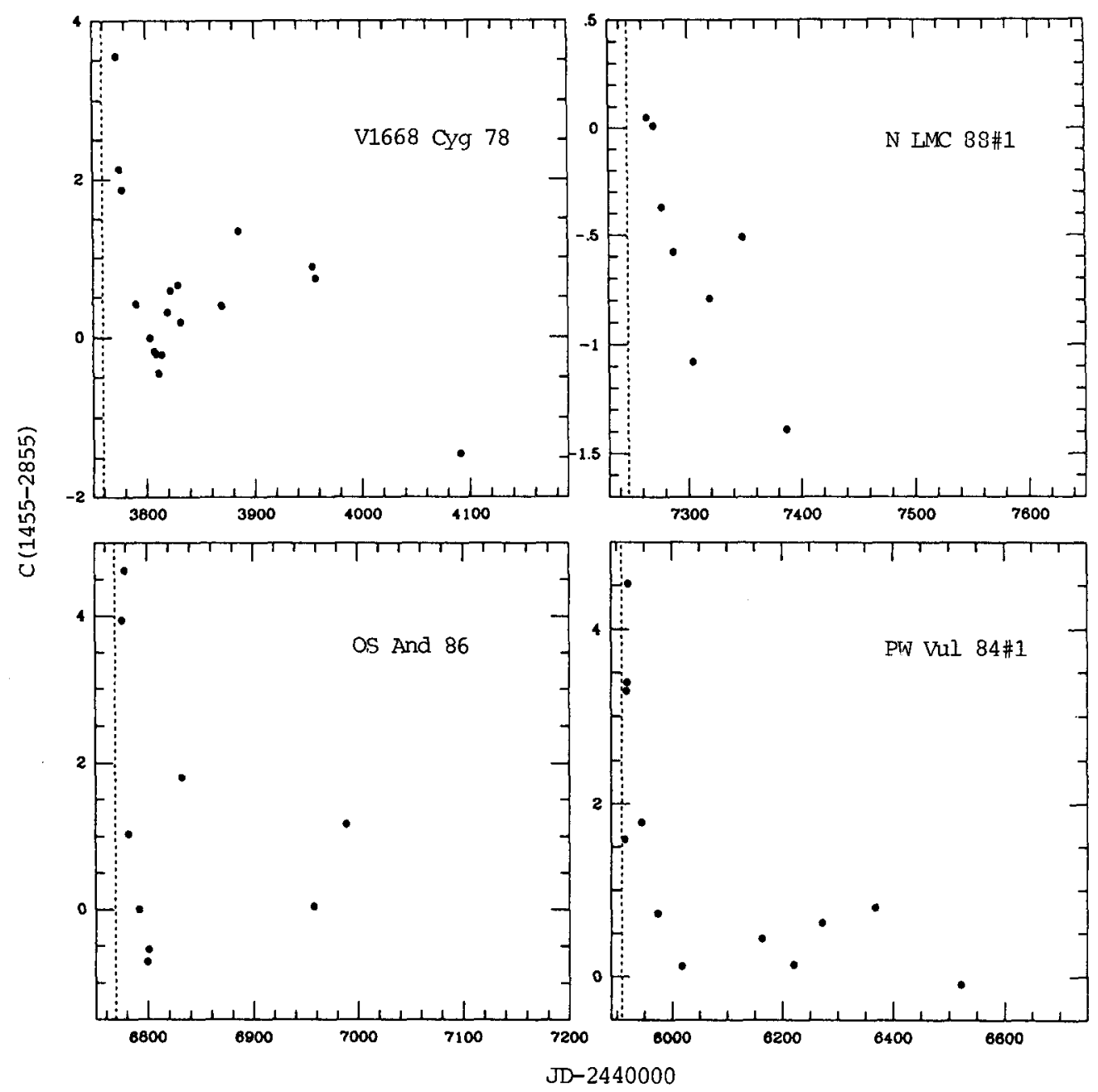

Fig. 5 Observed ultraviolet colour as a function of time for four novae monitored with IUE. See text. 

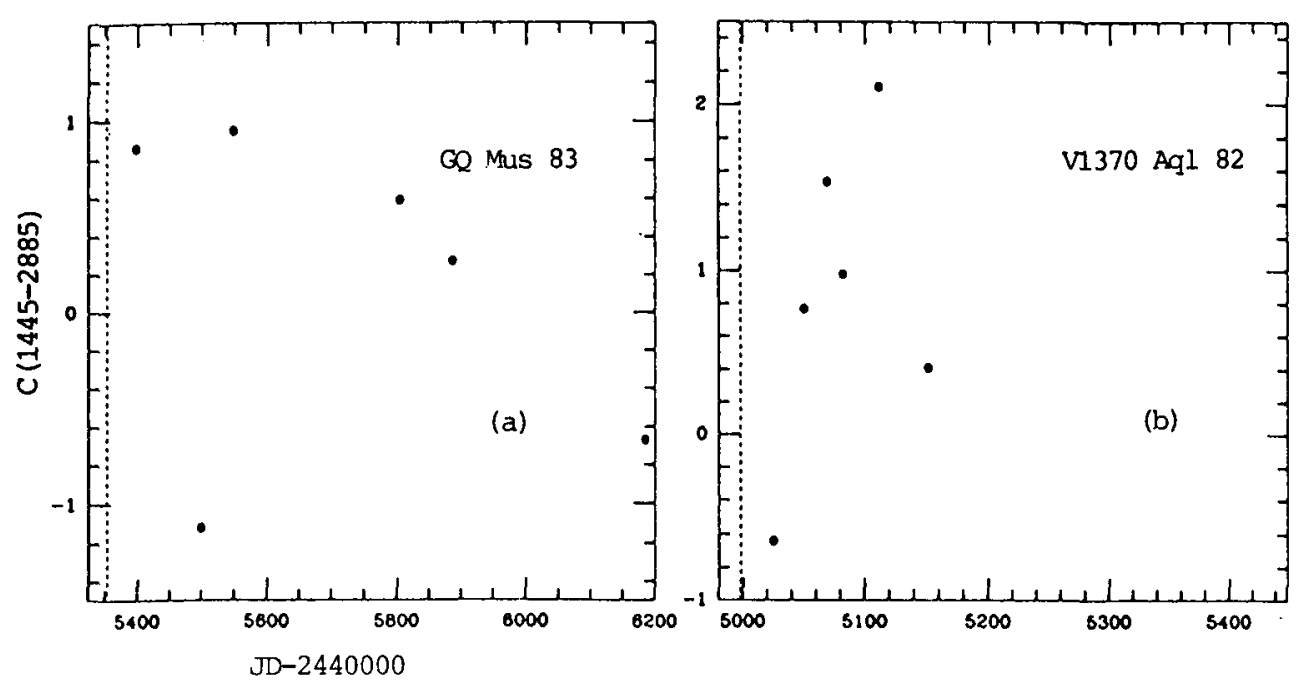

Fig. 6 Same as for Fig. 5 for GQ Mus 1983 and V1370 Aql 1982.

become hotter is recovered. The appearance of a relative maximum of the UV colour is easily understood in terms of an increasing amount of Balmer continuum (which contributes significantly to the 2855 A band) being produced through photoionization of the ejecta by the central source. This interpretation is corroborated by the fact that at this stage, the high ionization nebular lines become strong in the UV. After some time the emission measure in the nebular shell decreases so that the spectrum appears harder. In other objects, like PW Vul (see Fig. 5 ) and $Q U$ Vul the $U V$ colour do not show a minimum at intermediated stages like in the previous cases, but it decreases smoothly after maximum until it reaches a plateau denoting that, probably, the emission measure of the nebula never becomes large enough for its contribution to produce a change of trend in the UV colour development. Finally, we mention the peculiar cases of GQ Mus and V1370 Aql. The UV colour of GQ Mus (Fig. 6a) is characterized by an episodic hardening of the UV radiation near JD 5450 to be associated with the event discussed in sect. 2. In the case of v1370 Aql (Fig. 6b) the conspicuous reddening of the UV colour near JD 5100 is due to dust absorption, as discussed by snijders et al. (1987).

\section{The temperature of the remnants in recent novae}

One can easily estimate the temperature of the remnants of recent novae by applying the Zanstra method under the assumption that the nebula is optically thick to the stellar continuum shortward of $912 \mathrm{~A}$ (ionization of hydrogen) and 228 A (ionization of HeII) and that the ionizing continuum can be represented by a black body. The Zanstra HeII temperature in case $B$ recombination can be obtained from the ratio of the flux in the HeII 1640 A emission line (supposed to be optically thin) to the flux at 1445 A (supposed to represent the 

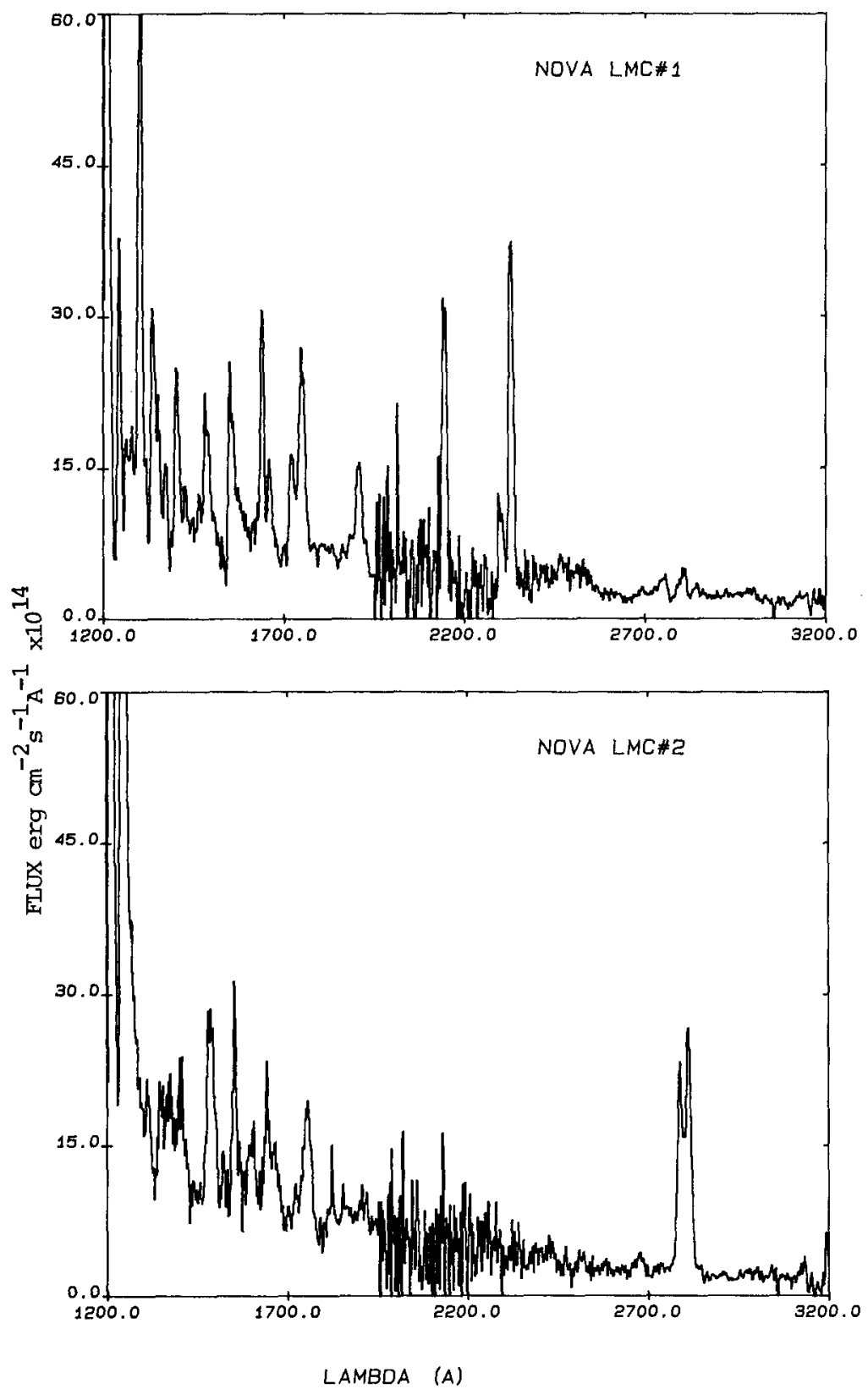

Fig. 7 Energy distribution of N LMC 1988\#1 observed on April 29, 1988 and N LMC 1988\#2 observed on December 29, 1988. The spectra are corrected for interstellar extinction. 
continuum from the hot source). Similarly, the hydrogen Zanstra temperature can be obtained from the ratio of continuum at $2855 \mathrm{~A}$ (supposed to be little contaminated by the hot continuum) to the flux at 1445 A (see e.g. Fernandez-Castro et al. 1988 for details). The temperatures derived from the two methods (which represent a lower limit to the temperature of the hot source) are generally consistent for the objects analysed. For N LMC 1988\#1 (observed in 1988, April 29 ) and N LMC 1988\#2 (observed in 1988, December 29) we find $T \sim 75000$ $\mathrm{K}$ and $70000 \mathrm{~K}$, respectively. The corresponding UV spectra of these novae are shown in Fig. 7. Larger values are found for V693 Cra $(\sim 100000 \mathrm{~K}$ in September 1981), V1668 Cyg 1978 (150000 K in Jan. 1980). It would be desirable to make a detailed investigation of how the temperature of the hot remnant changes with time for comparison with models.

\section{REFERENCES}

Bath. G.T., Harkness, R.P.: 1988, in "Classical Novae", ed. M. Bode, A. Evans, (John Wiley) p. 61

Cassatella, A., Selvelli, P.L.: 1988, in "A Decade of UV Agtronomy with IUE", GSFC, Greenbelt, ESA-SP-281, Vol. 1, p. 9

Evans, A.: 1989 , private communication

Fernandez-Castro, T., Cassatella, A., Gimenez, A., Viotti, R.: 1988, Ap. J. 324,1016

Friedjung, M.: 1988, in "Classical Novae", eds. M. Bode and A. Evans, (John Wiley) p. 187

Gehrz R.D.: 1988, Ann. Rev. Astron. Astrophys. 26, 377

Gallagher, J.S., Code, A.D.: 1974,Ap. J., 189,303

Hasgall, B.M.J. et al.: 1990, these Proceedings

Snijders, M.A.J., Batt, T.J., Seaton, M.J., Blades, J.C., Morton, D.C.: 1984 , Mon. Not. R. astr. Soc. $211,7 \mathrm{p}$

Snijders, M.A.J.: 1986, in "RS Ophiuchi", ed. M.F. Bode, VNU Science Press, p. 51

Snijders, M.A.J., Batt, T.J., Roche, P.F., Seaton, M.J., Morton, D.C., Spoelstra, T.A.T., Blades, J.C.: 1987, Mon. Not. R. astr. Soc. 228, 329

Starrfield, S.: 1986, in "New Insights in Astrophysics", University College London, ESA-SP263, p. 239

Starrfield, S. Sparks, W.M., Truran, J.W.: 1986, Astrophys. J. Letters 303 , L5

Sparks, W.M., Starrfield, S. W.M., Truran, J.W.: 1976, Astrophys. J. 208,819

Starrfield, S., Snijders, M.A.J.: 1987, in "Exploring the Universe with the IUE Satellite", Ed. Y. Kondo and W. Wamsteker, D.Reidel (Dordrecht), p. 377

Stickland, D.J., Penn, C.J., Seaton, M.J., Snijders, M.A.J., Storey, P.J.: 1981, Mon. Not. R. astr. Soc. 197, 107

Wamsteker, W.: 1979, Astron. Astrophys. 76, 226

Wamsteker, W., Driessen, C., Munoz, J.R., Hassall, B.M.J., Paisan, F., Barylak, M., Russo, G., Egret, D., Murray, J., Talavera, A., Heck, A.: 1989, Astron. Astrophys. Suppl. Ser. 79, 1

Williams,R.E., Ney, E.P., Sparks, W.M., Starrfield, S.G., Wyckoff, S., Truran, J.W.: 1985, Mon. Not. R. astr. Soc. 212,753 